\title{
Pertinencia y análisis del mercado para la toma de decisiones en instituciones de Educación Superior: Oferta de maestrías en Ciencias administrativas en la región Caribe Colombiana
}

\section{Relevance and market analysis for decision making in Higher Education institutions: Offer of Master's degrees in Administrative Sciences in the Colombian Caribbean region}

\author{
Leonardo Vassallo-Díaz* \\ Corporación Universitaria Americana - Colombia \\ ORCID iD: https://orcid.org/0000-0003-1901-6953 \\ lvassallo@coruniamericana.edu.co

\section{Stephanía Cárdenas-Herrera \\ Corporación Universitaria Americana - Colombia ORCID iD: https://orcid.org/0000-0002-4128-3735 \\ cardenasstephania@coruniamericana.edu.co}

Fecha de recepción: 26/04/2020

Fecha de evaluación: 20/05/2020

Fecha de aceptación: 19/07/2020

* Autor a quien debe ser dirigida la correspondencia

Cómo citar: Vassallo-Díaz, L., \& Cárdenas-Herrera, S. (2020). Pertinencia y análisis del mercado para la toma de decisiones en instituciones de Educación Superior: Oferta de maestrías en Ciencias administrativas en la región Caribe Colombiana. Revista Cientifica Anfibios, 3(2), 66-75. https://doi.org/10.37979/afb.2020v3n2.74

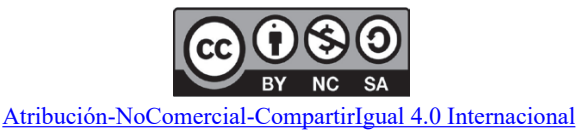

\section{Resumen}

El presente artículo científico recoge los resultados del estudio de pertinencia de la oferta de nuevos programas de maestría en Administración de Empresas en la Región Caribe y la demanda existente a través de un estudio transversal implementando un instrumento de recolección de información, a 396 participantes distribuidos en los 7 departamentos de la Región Caribe. La validación de la existencia de una demanda existente y la pertinencia de ofertar nuevos programas de maestría en el desarrollo sobre la base de los conceptos de pertinencia y demanda. Los resultados demuestran la pertinencia de la oferta de nuevos programas de maestrías en el área de Administración para la Región Caribe.

\section{Palabras clave:}

Pertinencia; demanda; educación superior; maestrías; administración.

\begin{abstract}
The present scientific article gathers the results of the study on the relevance of the supply of new master's programs in Business Administration in the Caribbean Region and the existing demand through a cross-sectional study implementing a information, 396 participants distributed in the 7 departments of the Caribbean Region. The validation of the existence of an existing demand and the relevance of offering new master's programs is developed on the basis of concepts of relevance and demand. The results demonstrate the relevance of the offer of new masters programs in the area of Administration for the Caribbean Region.
\end{abstract}

Keywords:

Relevance; demand; higher education; master programs; administration. 
Pertinencia y análisis del mercado para la toma de decisiones en instituciones de Educación Superior: Oferta de maestrías en Ciencias administrativas en la región Caribe Colombiana

\section{Introducción}

La formación de magísteres en la Región Caribe colombiana ha sufrido cambios significativos durante los últimos cinco años, lo cual impacta positivamente en los escenarios de desarrollo social y económico de la región y el país. En este sentido, el crecimiento de la oferta de posgrados ha permitido sostener un conjunto de transformaciones asociadas al crecimiento de empresas de base tecnológica, el perfeccionamiento de la capacidad de reacción delas empresas el aumento de capacidades dinámicas innovadoras en los distintos sectores de la economía, y un contexto deformación mucho más especializado y pertinente, elementos que han ido reconfigurando los sistemas de formación, producción y diversificación de cara a la enseñanza de tercer nivel.

Las reformas educativas en Colombia han provocado nuevas lógicas formativas durante el siglo XX, focalizadas en poner al estudiante como el centro del proceso de enseñanza-aprendizaje y en los resultados de aprendizaje (Decreto 1330 de 2019); sumado a esto, los cambios en la gestión del conocimiento científico y tecnológico en la segunda mitad del siglo XX, posibilitan la configuración de una sociedad cada vez más ajustada y sometida a la búsqueda de conocimiento y la información; Sin embargo, uno de los factores que ha tenido un avance poco significativo es la pertinencia del sistema educativo, en donde familias y los estudiantes encuentran una formación poco ajustada a las necesidades sociales y culturales de las regiones y la globalización, el mundo del trabajo, la vida en comunidad y la sociedad. Así, el rol del Estado y su grado de injerencia en este campo, integrando la participación de diversos proveedores educativos y se abrió un atractivo nicho de formación educativa ha impactado en la manera en cómo se plantea la educación y su impacto directo en el desarrollo integral de los ciudadanos. Además, en los últimos 5 periodos de gobierno, se estableció la necesidad de mecanismos de regulación, seguimiento y control de la calidad y la alta calidad de la formación en cabeza de la Comisión Nacional Intersectorial de Aseguramiento de la Calidad de la Educación Superior - CONACES y del Consejo Nacional de Acreditación - CNA, intensifican- do los esfuerzos por aumentar la equidad del acceso a la educación superior, lo que implicó un aumento de la matrícula en educación superior, amparado en créditos estatales y privados.

El objetivo principal del estudio es determinar el grado de pertinencia para el desarrollo de programas de Maestría en el contexto regional del Caribe colombiano, dirigido a la cualificación al más alto nivel de los actores del proceso formativo, profesional e investigativo que impacten la dinámica social, empresarial y económica de la ciudad y la región.

Lo anterior se desarrolló a partir de la identificación de la demanda potencial y expectativas del mercado acerca de la oferta de maestrías en las áreas de las ciencias administrativas y económicas y la determinación de las necesidades de los encuestados, respecto al interés académico en la oferta potencial de una nueva oferta de programas de maestría en la región caribe.

Este escenario de fortalecimiento de la oferta y acceso estudios superiores, permite evidenciar que los posgrados no se adaptan a las nuevas lógicas de funcionamiento del sistema universitario. El término pertinencia se entiende como algo que es oportuno, es decir que se ubica o realiza en el momento y espacio indicado. Cuando se realizan estos estudios es con el fin de dar a conocer la coherencia de los Programas Educativos en relación con el contexto y las necesidades demandantes de la sociedad. Es interesante, como los resultados del estudio de pertinencia y análisis de la demanda para la creación de programas de maestría en Administración revelan la verdadera línea de interés y la dinámica social y empresarial que necesita ser atendida de manera efectiva y eficiente, generando estados de sostenibilidad en los procesos y sustentabilidad en las soluciones que se plantean de cara a los retos de región y del país.

En primera instancia, se realiza un registro sobre tales programas en el país y se analiza su oferta a partir de información disponible en internet y una revisión documental. Un segundo momento gestiona, a partir de 396 encuestas en profundidad, la expectativa y aspiraciones 
de los potenciales estudiantes, se levantan las demandas que se realizan a estos programas.

\section{Marcos de referencia}

\section{Fundamentos teóricos}

Para el desarrollo de este artículo se escogió el concepto de pertinencia desarrollado por (Gibbons, 1998) basado en el documento "Pertinencia de la educación superior en el siglo XXI" (p. 4), el cual fue presentado en la Conferencia Mundial de Educación Superior, en donde propone que la educación es pertinente en la medida en que forme personas capaces de producir un conocimiento que respalde la economía y mejore las condiciones de vida de los ciudadanos, es por esto que la institución de educación superior deberá mostrar cómo contribuye al desarrollo de la sociedad. Por otro lado, nos encontramos con el planteamiento de (Malagon Plata, 2003), la relación con el sector productivo es una parte de la pertinencia; por esta razón, es importante tener claro también el concepto de pertinencia integral que "explique la relación teoría-práctica y la relación instituciones educativas-sociedad" (p. 24). Lo que quiere decir que la pertinencia debe ir relacionada de manera que demuestre cómo las IES atienden las necesidades y el mercado laboral y también en cómo inciden en los ámbitos social, político y cultural.

Asimismo, (Gottifredi A., 2000) afirma que "la pertinencia tiene relación directa con las expectativas y sensaciones de la sociedad con la universidad". La UNESCO había también definido la pertinencia como el "papel que desempeña y el puesto que ocupa la educación superior en el seno de la sociedad", pero también había considerado específicamente: "su misión y sus funciones, los programas, los modos de impartir la enseñanza y su contenido, como los temas de equidad, responsabilidad y financiamiento, al tiempo que destaca la libertad de cátedra y la autonomía institucional como principios sobre los que deben basarse todos los esfuerzos por garantizar y elevar la pertinencia" (UNESCO, Politicas para el Cambio y el desarrollo de la Educación Superior, 1995). Estas declaraciones reafirman el vínculo que debe tener la universidad y sociedad.
La educación no tiene como principal misión formar para un puesto de trabajo específico, lo importante es que pueda ayudar al desarrollo de las competencias correspondientes a un campo amplio en el ámbito laboral. (Narodowski, 2005) hace énfasis en que las mejoras del currículo deben realizarse en función de los problemas relativos al mundo del trabajo, de los avances tecnológicos y de la nueva configuración globalizada. "Las nuevas empresas valoran a un trabajador no por su conocimiento o información que se devalúa rápidamente sino por su sólida formación básica y el dominio de algunas aptitudes" (Ferraro, 1995).

Por su parte (Ramírez-Fernández, Machado-Licona \& Fernández-Ramírez, 2019) consideran que es importante que los profesionales sean formados con una nueva visión, con la capacidad de ser críticos y asumir los roles que las empresas y la sociedad demanden; en pro de lograr dichas capacidades en los profesionales es necesario que las Universidades acojan la calidad como principio rector de su gestión y fomenten la cultura de autoevaluación continua.

Para garantizar la calidad en la gestión Álvarez (2005) plantea que, "la mejora de la calidad implica cambios institucionales y de conducta de las personas; y, la evaluación exige autorregulación y por tanto la implicación de personas en el proceso de evaluación y mejora." De esta manera, la evaluación es un proceso que involucra a todos los estamentos de la IES ya que no se puede establecer acciones de mejora si no existe compromiso de las partes para realizar los cambios que se requiere mejorar. Los procesos de mejora de la universidad alcanzarán la calidad, cuando "participen o interactúen todos los atributos pertinentes" (Garduño, 1999).

La Universidad debe trabajar con una lógica que enfatice los valores humanos y "poniendo énfasis en la construcción de capacidades con profundo sentido ético, fundamentales en un mundo de rápidos cambios, gran volumen de información y la presencia de las tecnologías de la comunicación" (UNESCO, Conferencia Mundial sobre Educación Superior, La educación superior en el Siglo XXI: Visión y Ac- 
Pertinencia y análisis del mercado para la toma de decisiones en instituciones de Educación Superior: Oferta de maestrías en Ciencias administrativas en la región Caribe Colombiana

ción, 1998). Esto a su vez exige que haya una actualización permanente de la oferta académica de las carreras, garantizando siempre una formación que prepare y capacite al estudiante para que pueda contribuir con las competencias alcanzadas y enfrentar con eficiencia los retos del desarrollo social, económico y humano en un ámbito que es cada vez más competitivo.

"El diseño de un nuevo Plan de estudios debe estar apoyado por estudios y criterios que garanticen su pertinencia y factibilidad" (Lope \& Magaña, 2000), estos estudios deben mostrar que es necesario crear el nuevo proyecto y lo más importante que existen las condiciones para su desarrollo. Lo que quiere decir que los estudios de Pertinencia no deben verse como un simple trámite para continuar con el proceso de diseño, ya que son una pieza clave que permite enfocar el diseño.

En el Documento de Trabajo (UNESCO, Conferencia Mundial sobre Educación Superior, La educación superior en el Siglo XXI: Visión y Acción, 1998) agrega que "ser pertinente es estar en contacto con las políticas, con el mundo del trabajo, con los demás niveles del sistema educativo, con la cultura y las culturas, con los estudiantes y profesores, con todos, siempre y en todas partes". Lo que significa que debe existir una relación lógica entre lo que la sociedad espera de las instituciones y lo que éstas hacen.

Las instituciones de educación superior deben preocuparse por responder a las necesidades que demanda la sociedad. Una de las vías para cumplir con esta función es ofertar Programas Educativos que formen profesionales capaces de incidir en sus contextos.

(De Gottifredi, 2000), sostiene que "la evaluación del grado de pertinencia y de equidad de una institución universitaria requiere cuando menos que la institución se investigue a sí misma para descubrir cuál es la verdadera imagen que se construyó en el seno de la sociedad, de tal manera que, si ésta no es adecuada, se deberá trabajar para cambiarla. La equidad es la otra dimensión que debe controlarse con la puesta en marcha de nuevos proyectos educativos con el objeto de alcanzar una mayor cobertura de las prestaciones universitarias. El mayor desafío de la universidad del futuro es su activa participación en la discusión de las grandes problemáticas que afectan al tejido social aportando investigaciones objetivas, identificando problemas y sugiriendo alternativas para superarlos."

De manera que, en el espacio de la educación superior, la universidad es una institución que enseña pero que también aprende para transformarse a sí misma y poder intervenir en la transformación y mejora de la realidad. Así pues, la universidad enseña e investiga, transfiere conocimiento, y además se compromete en la transformación de la realidad.

\section{Factores de los que depende la Demanda Ed- ucativa}

Entre los factores que afectan la demanda educativa a nivel superior, de manera general se identifican aquellos factores que con mayor frecuencia influyen en el comportamiento de los individuos para acceder a la educación superior, como los que se señalan a continuación:

Factores demográficos están relacionados con el tamaño de la población y la estructura de las cohortes, la localización de los aspirantes, la dimensión social en el que se insertan y la condición económica y geográfica. Se estima que estos valores cobran gran importancia al momento de establecer la edad de egreso de la educación superior, en todos sus niveles, lo cual apunta a que se genere una expansión de la demanda educativa a niveles superiores (posgrados). Factores económicos, hacen parte de estos la economía vinculada al gasto público más exactamente el relacionado con la inversión en educación; a la par se encuentra también la inversión que deben asumir las personas para tener acceso a la educación. Factores sociales y familiares, incluyen la formación de los padres y la importancia que los mismos otorgan a la educación de sus hijos, así como el entorno donde se da el proceso de formación ya sea en una ciudad o en el campo. Factores Individuales, se vinculan con la habilidades y capacidades del individuo como el intelecto, las competencias, las habilidades, recursividad y capacidades propias adquiridas mediante su 
experiencia en la educación secundaria, como complemento a estos factores se puede mencionar el estado psicológico que puede llevar al individuo a estar desmotivado o motivado para continuar su estudio en el nivel de educación superior.

De otra parte, los factores Institucionales tienen que ver con los rasgos propios de las instituciones de educación superior entre estos se encuentra el reconocimiento, su trayectoria, el reconocimiento de la marca, entre otras.

Fueron objeto de esta investigación determinar algunos de los factores mencionados anteriormente desde el aspecto familiar, individual, Institucional y económico.

\section{Metodología}

El tipo de investigación implementado se ha determinado como descriptivo comparativo y correlacional de acuerdo con los planteamientos de (Méndez, 2003), pues el tipo de estudio descriptivo “.... identifica características del universo investigado". Del mismo modo, (Tamayo y Tamallo, 2008), establece que esta “... trabaja sobre realidades de hecho y sus características fundamentales es presentarnos una interpretación correcta". Comprende la descripción, registro, análisis e interpretación de la naturaleza actual y la composición o procesos de los fenómenos como es el caso del presente estudio que tiene como propósito determinar la pertinencia de crear un programa de maestría de Administración de Empresas para ofertar en la Región Caribe y analizar su posible demanda.

El presente estudio es considerado de tipo descriptivo, ya que sólo pretende recolectar información real acerca del tema de investigación para describir las variables de marketing interno y calidad de servicio, identificando sus características, dimensiones, técnicas utilizadas, formas de manifestación, entre otros. (Hernández, Fernández, \& Baptista, 2003) señalan que la investigación descriptiva consiste en describir fenómenos, situaciones, contextos y eventos; esto es, detallar cómo son y cómo se manifiestan. Según (Méndez, 2008), su propósito “es la delimitación de los hechos que conforman el problema de investigación".
Desde el punto de vista de (Tamayo y Tamayo, 2001), la investigación descriptiva “comprende la descripción, registro, análisis e interpretación de la naturaleza actual. Y la composición o procesos de los fenómenos. El enfoque se hace sobre conclusiones dominantes o sobre cómo una persona, grupo o cosa se conduce o funciona en el presente.

Esta caracterización de los sujetos de investigación estuvo apoyada en un estudio de campo de las necesidades que los profesores, administrativos y egresados de las distintas especialidades de IES de la Región Caribe, consideran relevantes en la actualidad para incrementar su desarrollo y cualificación al más alto nivel.

Los datos fueron suministrados de una muestra de 396 encuestados, conformada en su gran mayoría por personas que se desempeñan como profesionales en ejercicio y un número significativo de recién egresados, o con titulación, de pregrado y especialistas. Los datos fueron recogidos para explicar la realidad en relación con las intenciones e intereses por realizar estudios de maestría en Administración. Finalmente se realiza una etapa de investigación concluyente descriptiva inmersa en el campo cualitativo, con el apoyo de técnicas estadísticas para el análisis y proceso de la información de los datos y obtener conclusiones fiables que soporten la factibilidad de programas de maestría en el área de ciencias administrativas con asentamiento en Barranquilla y abierta a la Región Caribe.

Como resultado de este estudio se espera presentar al Ministerio de Educación Nacional el diseño de los programas de maestría en Administración, para su estudio y futura inclusión entre las ofertas de oportunidades de estudio para profesionales y académicos, en la ciudad, la región y el país.

\section{Análisis de los resultados}

\section{Integración de los elementos propios de la recolección y análisis de las fuentes prima- rias para el estudio.}

Tras la recolección de información a través del análisis documental realizado y presentando previamente para determinar las preferen- 
Pertinencia y análisis del mercado para la toma de decisiones en instituciones de Educación Superior: Oferta de maestrías en Ciencias administrativas en la región Caribe Colombiana

cias de la población estudiantil, se recurrió a grupos focales para establecer intencionalidad de adelantar estudios de maestría y sus correspondientes estrategias dirigidas a la apertura de este tipo de programas.

Respecto a los resultados obtenidos, su descripción e interpretación al instrumento empleado se pueden conocer las siguientes situaciones: La edad promedio de los encuestados es entre 39 y 40 años. El 62\% es casado, el 28\% es soltero y el 5\% vive en unión libre. El 38\% nació en Barranquilla, el 18\% nació en Cartagena y el otro $18 \%$ nació en otro municipio del país. Es decir, más de un $50 \%$ de la muestra hace parte directa del contexto Caribe.
Los departamentos de mayor procedencia de los encuestados son Atlántico (47\%), Bolívar (26\%) y La Guajira (11\%). El 48\% de los encuestados reside en Barranquilla, el 23\% reside en Cartagena, el 8\% reside en Riohacha y el $8 \%$ reside en otro municipio.

El 19\% de los encuestados tiene título de administrador de empresas, el $14 \%$ es economista, $13 \%$ es contador público. En total, el $56 \%$ de los encuestados tiene un título profesional en ciencias económicas administrativas y contables, área de conocimiento en la que se circunscriben las posibles maestrías a ofertar.

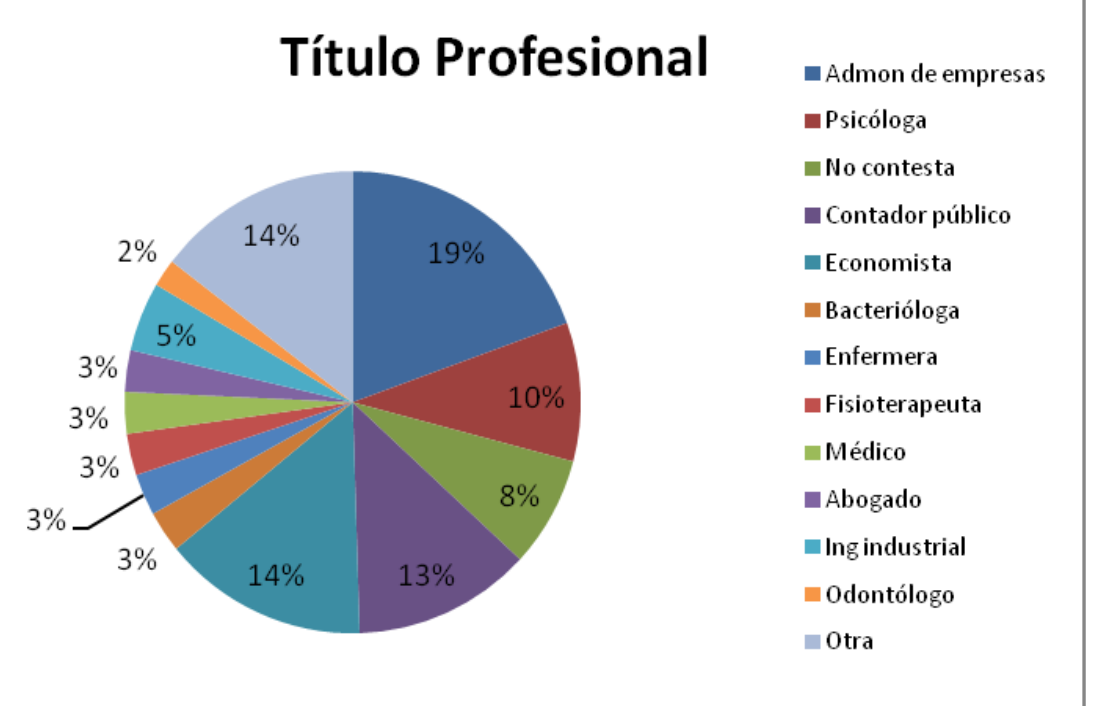

Figura 1. Título profesional de los encuestados.

Fuente: Elaboración Propia

Las universidades que mayor representación tienen entre la muestra encuestada es la Universidad Simón Bolívar (22\%), seguida de la Autónoma del Caribe (19\%) y la Corporación Universitaria Americana (19\%), siendo un termómetro importante la ciudad de Barranquilla para el estudio realizado.

El 53\% de los encuestados trabaja en una entidad privada, el 38\% trabaja en una entidad pública, el 9\% no informa.

El $42 \%$ de los encuestados trabaja en una Universidad, el $31 \%$ trabaja en un tipo de entidad dedicada a actividades diferentes a la educación, el 16\% trabaja en una institución uni- versitaria. En total, el $61 \%$ de los encuestados trabaja en Educación Superior, un segmento de mercado, donde la demanda de maestrías para estos profesionales es mayor que en otros sectores laborales, siendo pertinentes con los posibles interesados en la oferta que se daría apertura.

De la muestra tomada, el $46 \%$ de los encuestados se dedica a actividades administrativas, el $29 \%$ se dedica a la docencia, el 14\% lleva alguna actividad directiva y el $11 \%$ se dedica a actividades investigativas. Si sumamos los docentes y los que se dedican a la investigación, obtenemos que un $40 \%$ de la muestra pertenece a un posible nicho de mercado interesado en la apertura de dicha oferta. 


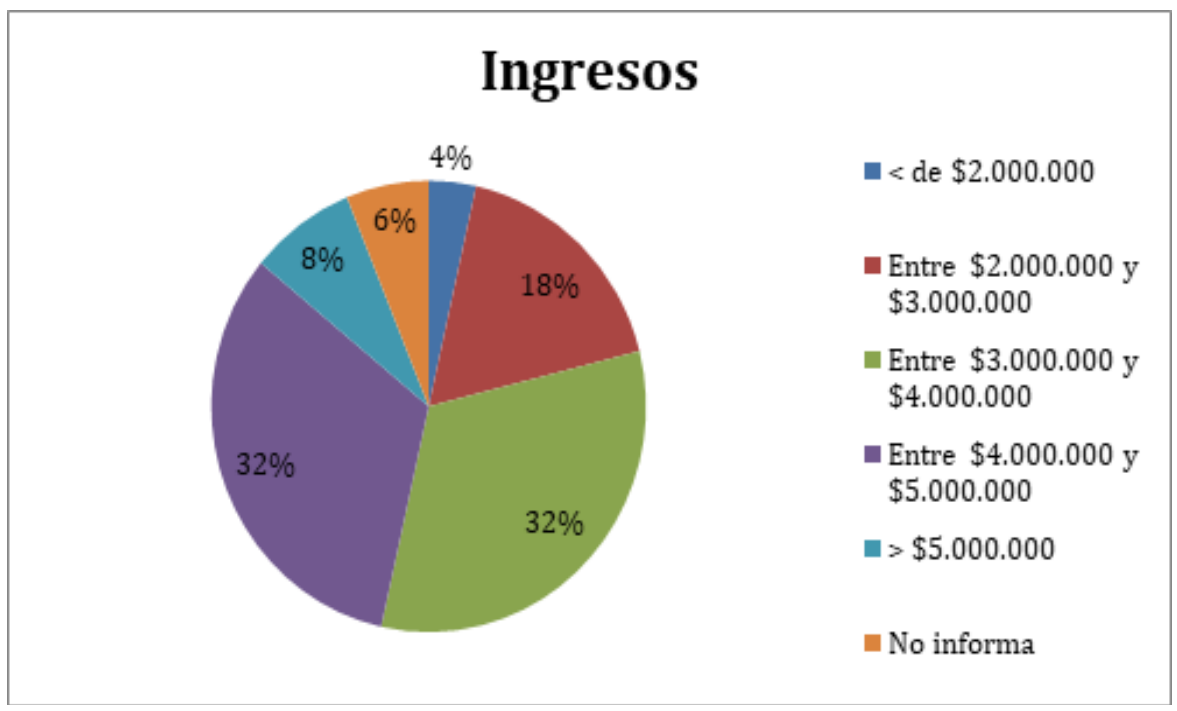

Figura 2. Ingresos mensuales de los encuestados.

Fuente: Elaboración Propia

El 18\% de los encuestados gana entre uno y dos millones de pesos, el $32 \%$ gana entre dos y tres millones de pesos, el $32 \%$ gana entre tres y cinco millones de pesos, el $8 \%$ gana más de cinco millones de pesos. Esto nos permite deducir que el $40 \%$ de la muestra tiene ingresos superiores a los $\$ 3.000 .000$, un factor de mucho interés al momento de establecer un valor adecuado de mercado para satisfacer la posible demanda del programa universitario que se analiza en este estudio. Del estudio, se obtuvo que el $88 \%$ de los encuestados tiene interés en realizar una maestría en el área de su preferencia, el 17\% lo realizaría inmediatamente, el $27 \%$ lo haría en un año y el $44 \%$ lo haría en el mediano plazo. Con esto, confirmamos la demanda latente del mercado en la Región Caribe por programas de maestría.

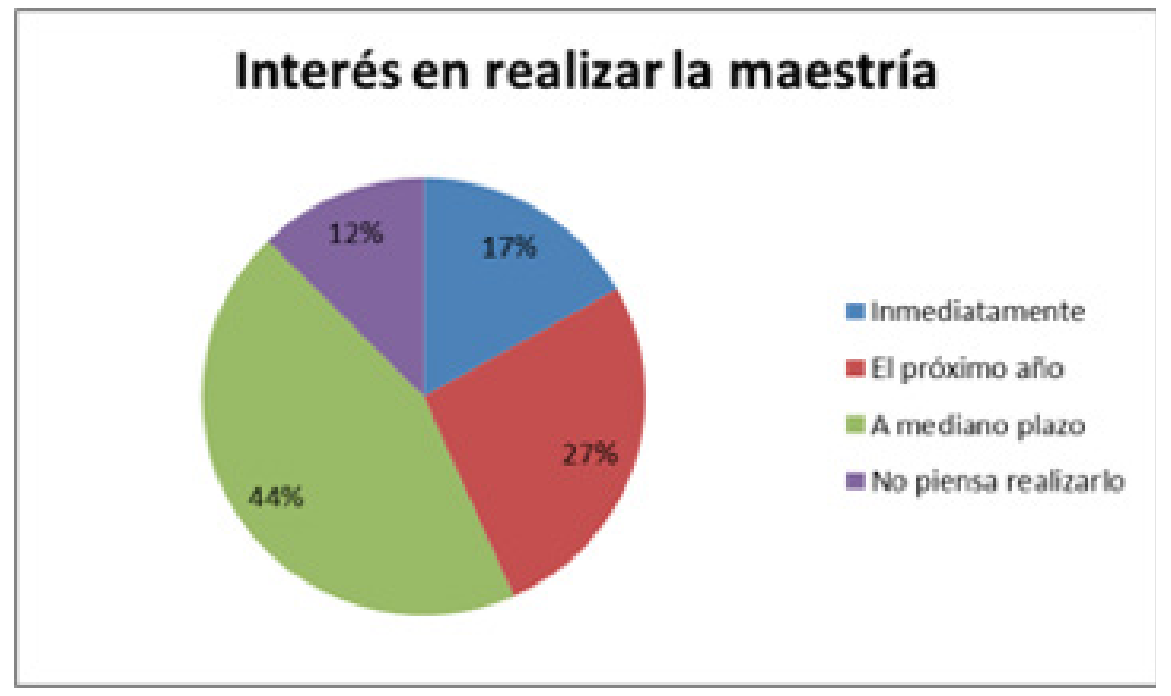

Figura 3. Interés en cursar una maestría.

Fuente: Elaboración Propia

Al indagar sobre el interés en realizar una maestría, el $29 \%$ de los encuestados piensa que una maestría le permitiría avanzar en su desarrollo personal, el $27 \%$ piensa que le serviría para mejorar su posición laboral, el 14\% piensa que le permitiría conseguir un nuevo hori- zonte laboral. Dentro de los datos captados en el estudio, al medir la periodicidad más conveniente para realizar alguna maestría para la muestra del estudio, el análisis de datos arrojó que los encuestados prefieren que se impartan las clases de forma mensual para los encuesta- 
Pertinencia y análisis del mercado para la toma de decisiones en instituciones de Educación Superior: Oferta de maestrías en Ciencias administrativas en la región Caribe Colombiana

dos de la muestra tomada, teniendo específicamente, una semana de trabajo (58\%). Esta premisa debe tenerse en cuenta al momento de revisar la carga académica del programa y los tiempos a desarrollarlo, de modo que se pueda integrar un contenido acorde a las necesidades de conocimiento del mercado laboral con la periodicidad adecuada para ello.

Cómo jornada deseada para desarrollar sus estudios, los encuestados prefieren la franja tarde-noche para cursar una maestría $(63 \%)$, teniendo como factores que llevan a la escogencia de esta, los tiempos que destinan en la jornada de la mañana a sus profesiones.

Siempre, en una sociedad tan desigual y con tantos inconvenientes económicos como la que encontramos actualmente en Colombia, es un punto importante, antes de lanzar una oferta al mercado, conocer si los valores se acomodan a la capacidad adquisitiva del público y establecer qué mecanismos de pago y financiación serán los utilizados por estos, para poder acceder al servicio ofertado. Al indagar en el estudio sobre las fuentes de financiación, los encuestados manifestaron que, el $29 \%$ de ellos pagaría su matrícula con recursos propios, el $19 \%$ usaría créditos bancarios, el $18 \%$ usaría créditos con el ICETEX, el 12\% solicitaría crédito directo con la institución y el $22 \%$ solicitaría apoyo institucional a nivel de becas/ beneficios para poder realizar dicho estudio.

Se hace necesario, establecer unas fuentes de financiación de fácil acceso para los aspirantes, ya que más del $45 \%$ de la muestra manifiesta que hará uso de este tipo de formato para poder acceder a la oferta educativa propuesta.

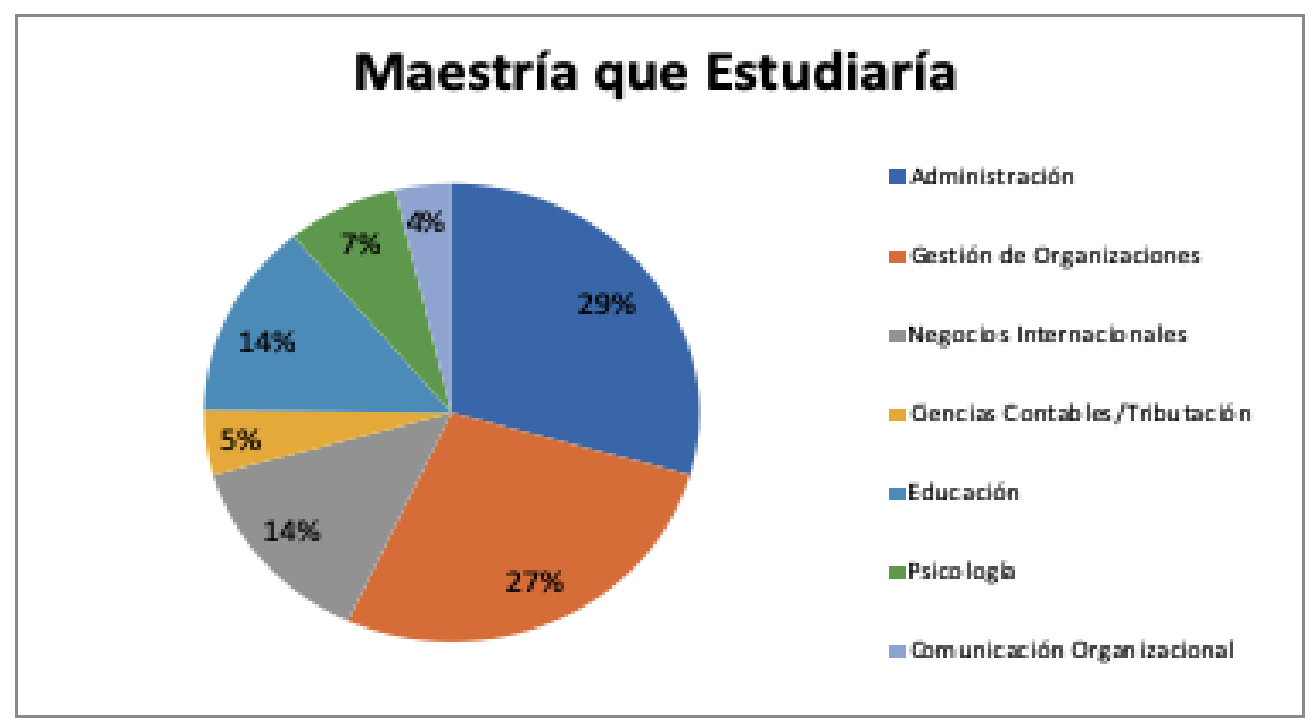

Figura 4. Áreas de Interés de los encuestados por estudios de maestría en la Región Caribe. Fuente: Elaboración Propia

En el ítem correspondiente a la consulta sobre el área de estudio en el cual realizaría dicho estudio encontramos que, el 29\% de los encuestados probablemente haría una maestría en Administración, el $27 \%$ lo haría en Gestión de organizaciones, siendo el campo administrativo/organizacional el que mayor interés demanda por parte de la muestra encuestada. Otro grupo significativo de encuestados (14\%) se inclinaría por estudios de maestría en el área de los Negocios Internacionales y otro $14 \%$ tomaría una maestría en Educación. Con menor nivel de preferencia para la muestra, pero con gran potencial de demanda por el cubrimiento de necesidades regionales en el área, se encuentran los programas de maestría en Psicología con un 7\%, Ciencias Contables 5\% y Comunicación Organizacional con un 4\%.

Se evidencia, con el estudio aplicado, que existe una demanda activa para la oferta de maestrías en la Región Caribe, siendo notorio también que el área de Administración, genera interés para los habitantes de nuestra región, los cuales estarían dispuestos a cursar un estudio de tercer nivel para mejorar sus condiciones laborales y avanzar en su campo dentro del mercado profesional de la región y del país. 


\section{Conclusiones}

El objetivo principal del estudio se basó en determinar la pertinencia para el desarrollo y oferta de nuevos programas de Maestría en el área de Administración en el contexto regional del Caribe colombiano, y la demanda existente en el mercado para la comercialización de las mismas.

Evidenciado lo anterior se concluye dando respuesta a que, si es pertinente la presentación del diseño de programas de Maestría en Administración en la Región Caribe para cubrir las necesidades y expectativas que esta requiere, tendiente a ampliar la oferta curricular a los estudios para graduados y proporcionar a los egresados los medios científicos y prácticos, para que puedan generar nuevos conocimientos en esta disciplina. Como resultado, la investigación se enmarca en el tipo de proyecto, basada en necesidades detectadas en el trabajo de campo.
Los aspectos que corroboran lo expresado se evidencian en los resultados del estudio realizado, en el que el $88,67 \%$ de los encuestados para esta investigación son conscientes de la realidad en concordancia con sus necesidades y las del área geográfica donde habitan, en tal virtud estarían dispuestos a disponer de recursos económicos para adelantar estudios de maestría en este campo específico de la gestión.

Resulta favorable detectar, además, en el estudio que, el $67.3 \%$ de los encuestados cuentan con estudios a nivel de especialización, lo que permite pensar que esta población es la más directamente referenciada para aprovechar la oportunidad de iniciar estudios en programas de maestría en los próximos años. Además, es destacable el programa y el área de Administración de Empresas como la opción escogida por el $29 \%$ de los encuestados, siendo la tendencia más alta de todas y corroborando la demanda existente para el nuevo programa.

\section{Referencias}

Aparicio Izquierdo, F., \& Gonzalez Tirado, R. (1994). La calidad de la enseñanza superior y otros temas universitarios. Madrid: ICE de la Universidad Politpecnica.

ASCUN. (1997). Hacia una Agenda de la Educación Superior en Colombia. ASCUN.

Barrón Tirado, C. (2000). Formación en competencias y certificación profesional. (25), 17-44.

Bovill, C., Morss, K., \& Bulley, C. (1009). Should students participate in curriculum design? Discussion arising from a first year curriculum design project and a literature review. Pedagogic Research in Maximising Education.

De Alba, A. (1991). Evaluación curricular: conformación conceptual del campo. México: UNAM.

De Gottifredi, N. (2000). Una forma de profundizar la relación universidad - sociedad. Facultad de Humanidades y Ciencias Sociales.

Ferraro, R. (1995). Educados para competir. Buenos Aires: Sudamericana.

Garcia Guadilla, C. (1996). Situación y proncipales dinámicas de transformación de la educación superior en América Latina. Caracas: CRESALC / UNESCO.

Garduño, L. (1999). Hacia un modelo de evaluaciónde la calidad. Iberoamericana de Educación, 103.

Gibbons, M. (1998). Pertinencia de la educación superior en el siglo XXI. Documento presentado como una contribución a la Conferencia Mundial sobre la Educación Superior de la UNESCO.

Gottifredi A., N. (2000). Una forma de profundizar la relación universidad - sociedad. Argentina: Cuadernos de la Facultad de Humanidades y Ciencias Sociales. Universidad Nacional de Jujuy, (15), 31-41. 
Pertinencia y análisis del mercado para la toma de decisiones en instituciones de Educación Superior: Oferta de maestrías en Ciencias administrativas en la región Caribe Colombiana

Hernandez, R., Fernandez, C., \& Baptista, P. (2003). Metodología de la investigación. Mc Graw Hill, 6 edición.

Lope, F., \& Magaña, M. (2000). Pertinencia y factibilidad de planes y programas de Estudio. En ANUIES (Ed.), Evaluación del Desempeño del personal académico. Análisis y propuesta de metodología básica. México: ANUIES.

Malagon Plata, L. A. (2003). La pertinencia en la educación superior. Elementos para su comprensión. Educación Superior. Vol. XXXII (3), Núm. 127.

Mendez, C. (2003). Metodología de la investigación. In Metodología de la investigación (p. 137).

Mendez,C. (2008). Diseño y desarrollo del proceso de investigación con énfasis en ciencias empresariales.

Narodowski, M. (2005). Notas para comprender el currículo escolar en la actualidad. Novedades Educativas, Año 18 No. 180.

Nation, I., \& Macalister, J. (2010). Language Curriculum Design. New York \&. London: Routledge Organización de la Naciones Unidas para la Educación la Ciencia y la Cultura (1990). Declaración Mundial de la Educación Superior. From http://unesdoc.unesco.org/images/0011/001163/116345s.pdf

Ramírez-Fernández, R., Machado-Licona, J., \& Fernández-Ramírez, O. (2019). Calidad en la educación universitaria, desde el programa de ingeniería de sistemas: una visión cualitativa de la educación superior. Revista Científica Anfibios, 2(2), 41-50. https://doi.org/10.37979/afb.2019v2n2.49

Tamayo y Tamallo, M. (2008). Investigación científica.

Tamayo y Tamayo, M. (2001). El proceso de la innvestigación. Ediciones Limusa Tercera edición.

UABJO. (2017). Modelo Educativo. Oaxaca: Universidad Autónoma “Benito Juárez” de Oaxaca.

UNESCO. (1990). Declaración Mundial de la Educación Superior. UNESCO. From http://unesdoc.unesco.org/images/0011/001163/116345s.pdf

UNESCO. (1995). Politicas para el Cambio y el desarrollo de la Educación Superior. Caracas: UNESCO.

UNESCO. (1998). Conferencia Mundial sobre Educación Superior, La educación superior en el Siglo XXI: Visión y Acción. París: UNESCO.

Vessuri, H. (2008). De la pertinencia social a la sociedad del conocimiento. En La educación superior en América Latina y el Caribe: diez años después de la Conferencia Mundial de 1998. Pontificia Universidad Javeriana. IESALC - UNESCO. 